\title{
Non-Orthogonal Resource Sharing Optimization for D2D Communication in LTE-A Cellular Networks: A Fractional Frequency Reuse-Based Approach
}

\author{
Devarani Devi Ningombam and Seokjoo Shin * \\ Department of Computer Engineering, Chosun University, 309 Pilmun-daero, Dong-gu, Gwangju 61452, Korea; \\ devaraninin@gmail.com \\ * Correspondence: sjshin@chosun.ac.kr; Tel.: +82-62-230-6030
}

Received: 20 August 2018; Accepted: 3 October 2018; Published: 5 October 2018

\begin{abstract}
To handle the fast-growing demand for high data rate applications, the capacity of cellular networks should be reinforced. However, the available radio resources in cellular networks are scarce, and their formulation is expensive. The state-of-the art solution to this problem is a new local networking technology known as the device-to-device (D2D) communication. D2D communications have great capability in achieving outstanding performance by reusing the existing uplink cellular channel resources. In D2D communication, two devices in close proximity can communicate directly without traversing data traffic through the evolved-NodeB (eNB). This results in a reduced traffic load to the eNB, reduced end-to-end delay, and improved spectral efficiency and system performance. However, enabling D2D communication in an LTE-Advanced (LTE-A) cellular network causes severe interference to traditional cellular users and D2D pairs. To maintain the quality of service (QoS) of the cellular users and D2D pairs and reduce the interference, we propose a distance-based resource allocation and power control scheme using fractional frequency reuse (FFR) technique. We calculate the system outage probability, total throughput and spectrum efficiency for both cellular users and D2D pairs in terms of their signal-to-interference-plus-noise ratio (SINR). Our simulation results show that the proposed scheme reduces interference significantly and improves system performance compared to the random resource allocation (RRA) and resource allocation (RA) without sectorization scheme.
\end{abstract}

Keywords: device-to-device communication; fractional frequency reuse; outage probability; throughput; spectral efficiency

\section{Introduction}

Device-to-device (D2D) communication underlaying long term evolution-advanced (LTE-A) cellular networks is proposed as an up-and-coming technology for next-generation cellular networks (5G). The first standardized exertion related to D2D communication has been proposed in LTE Release 12, in which devices in close proximity to each other can communicate directly bypassing the evolved-nodeB (eNB), thus providing new commercial and public safety proximity services. To date, D2D communication has gained significant attention over traditional cellular communication owing to its ability to fulfil the ever increasing user demand for higher data rate, reduced mobile data traffic, reduced end-to-end delays, higher system spectrum efficiency and lower power consumption. Integrating D2D communications with traditional cellular networks can resolve the problem of available spectrum discovery and avoidance of collision between D2D pairs and cellular users [1]. In a D2D communication underlaying a cellular network, D2D pairs reuse the licensed cellular band to improve system spectrum efficiency, which leads to imminent interference between cellular users 
and D2D pairs, and also among the D2D pairs. To mitigate the interference caused while reusing frequency bands by the D2D pairs, investigation on the random resource allocation (RRA) method has been proposed in the literature [2]. In the paper, the available D2D pairs can reuse any available channel resources of the cellular users and derived a closed-form expression for the outage probability. In other words, this paper controlled uplink interference by keeping a minimum distance between users. However, in this study, efficient utilization of the available frequency bands was not achieved. A low-complexity power allocation problem for D2D communications has been formulated in [3]. The results showed that their proposed scheme improves the throughput. However, in their study, the whole frequency band was not used. Therefore, the proposed scheme was not spectrally efficient. The fractional frequency reuse (FFR) method is a well-known technique to mitigate interferences caused by the frequency reuse method. FFR is radio resource partition method in the LTE-A system, which partitions the cell's available band into different sections such that the users of the adjacent cell do not interfere with each other while reusing the cellular frequency by the D2D pairs [4]. In [5], the authors proposed a joint resource block and power control method by using Nash Equilibrium computation technique. The results showed that their proposed scheme minimizes interference. However, optimal sum rate was not achieved. In order to improve spectrum efficiency, the authors in [6] proposed a context-aware cluster based D2D communications. The disadvantage of the proposed method is that the network model increases the interference.

The rest of the paper is structured as follows: contributions of the proposed scheme are listed in Section 2. Section 3 presents the related works and Section 4 gives an overview of the D2D communication, explaining the cell sectorization and FFR scheme comprehensively. Sections 5 and 6 present the network model and problem formulation, respectively. Similarly, Sections 7 and 8 illustrate the system throughput optimization formulation and channel model, respectively. Section 9 presents computational complexity and use case scenario. Finally, Section 10 presents performance evaluation and Section 11 concludes the paper.

\section{Contribution}

Inspired by the existing contributions in literature, we proposed a new radio resource allocation and power-control scheme for network-assisted D2D communications underlaying the uplink multicell cellular network. In this paper, we considered the FFR scheme with the cell-sectorization method. In the FFR scheme, the cell coverage region is divided into two non-overlapping regions, namely the inner cell region near the eNB and the outer cell region that covers the edge of the cell. In the sectorization method, the subdivided cell regions are partitioned into three sections with different frequency sub-bands using three $120^{\circ}$ directional antennas. In the proposed FFR scheme, the D2D pairs in the inner cell regions can only reuse the available cellular links of the outer cell regions and vice versa, and one cellular link can be reused at maximum by one D2D pair. This will make efficient utilization of most of the available resources in each section of a cell. The resource reusing phenomenon is driven by the channel co-efficients between cellular users and D2D users and between eNB and both the users. In this paper, we considered the distance of the devices from the eNB to reduce the interference from the cellular users to the D2D pairs.

We proposed different scenarios: the D2D pairs located in the same cell region, D2D pairs located in the different cell regions and varying the number of D2D pairs with respect to the cellular users. The performances are compared with the random cellular resource reuse by the D2D pairs. We formulate the optimization problem, which aims at maximizing the total system throughput limited to the knowlege of target signal-to-interference-plus-noise ratio (SINR) and power level. The results show that the proposed resource allocation and power-control scheme using the FFR method improves the system performance in terms of system throughput and spectrum efficiency of the cellular network by mitigating the interference introduced by the D2D users in the network. 


\section{Related Works}

The D2D network underlaying a cellular network that reuses the traditional cellular links is presented in [7]. It has been shown that the performance of the cellular network is affected by the interference caused by D2D communication. The work in [8] proposed a post-resource allocation and power control scheme for cellular users. Frequency reuse scenario is determined based on the interference generated by the D2D users. The disadvantage of this study is that an omnidirectional antenna was considered, which increases the interference in the system. In [9], system capacity evaluation using a blocking queuing model have been presented. In their network, an overlay D2D communication mode is discussed and formulated a fair admission control problem. Results showed that their proposed scheme improves the system throughput. The disadvantage of the scheme is that the queueing model is not assumed precisely. Network-assisted D2D communication and its advantages are listed in [10]. In [11], a two-step approach for D2D communication underlaying uplink cellular network has been discussed. At the first-step: a novel resource allocation based on the static transmit power was analyzed. At the second-step: interference minimization problem was formulated for dynamic reusing of cellular resources. However, in their study, quality of service (QoS) analysis was not considered.

The FFR is defined as the rate at which the same frequency can be used by the cellular users and D2D pairs simultaneously in a cellular network. In literature, studies have been conducted on FFR for interference cancellation and reduction for cellular users [12]. The work in [13] proposed a virtual cell sectorization method to jointly enhance resource allocation and reuse in a network-controlled D2D communication. Frequency reuse scenario is analyzed in terms of the maximum spatial distance. Comparing the result of the proposed method with that of the random resource allocation scheme shows the enhancement in system capacity. In [14], the authors proposed a frequency-reuse method by considering different transmission powers of cellular users and D2D pairs based on the user's accessing location. The authors demonstrate that their proposed scheme provides an improvement in the D2D pairs and cellular users as compared to the random resource-allocation method. The cellular system throughput optimization problem is formulated in many studies in the literature. The authors in [15] proposed an optimization problem that considers QoS constraints on the cellular users and D2D pairs. In their study, the optimal conditions for power for each subchannel was analyzed. Also, a dual-based iterative algorithm was derived to minimize the interference level. However, the introduced scheme is not spectrally efficient. In [16], the authors proposed a resource-allocation scheme for D2D pairs by dividing whole cell area into two non-overlapping regions, without cell sectorization (RA w/o sectorization) scheme. Uplink interference from the D2D pair to the base station, and also the interference from cellular users to the D2D pairs were analyzed in their study. It has been shown that reusing more than one channel resources of cellular users increases the system throughput significantly. The disadvantage of the study is that the network model is not analyzed for densely allocated users. In [17], the authors presented a D2D communication with FFR and fractional power-control schemes. The paper focused on the coverage region for both the D2D pairs and cellular users based on transmitter power. However, the paper does not consider the resource-reuse method among cellular users and D2D pairs. The D2D communication as the underlay to the orthogonal frequency division multiple access (OFDMA) is analyzed in [18]. In the paper, the authors presented a downlink resource-reuse method that considers only the outer cell region users to avoid interference from the eNBs. Dynamic power control for D2D communications underlaying uplink multi-cell networks has been presented in [19]. In their work, an optimization problem is formulated to minimize the interference caused by the D2D users to the conventional cellular users. The results showed that their proposed method the achieves higher system throughput.

\section{Overview of Device-to-Device Communication}

The difference between traditional cellular networks and D2D communications is, in a traditional cellular network a device can not directly communicate with each other and all communications are 
carried out through the eNB. To establish a D2D communication among the devices in close proximity to each other, there are two main mechanisms are involved. First, the peer device discovery and second, the D2D session setup. Peer device discovery can be subdivided into restricted-peer device discovery and open-peer device discovery. In restricted-peer device discovery, the device needs certain permissions to access the network, thus maintaining the user's privacy. For open-peer device discovery, the device can be identified as long as it is near another device. The device session setup initiates when the device-peer discovery is finished. The device session setup is subdivided into two modes. First, the IP-based detection mode and second, the dedicated D2D signaling. In the IP-based detection mode, the packet data network (PDN) gateway reveals the IP header of the data packets, thus the eNB maintains control over the D2D pair. For dedicated D2D signaling, dedicated D2D session setup architecture is accomplished. This reduces the load to the eNB and results in a faster system. According to the link establishment, D2D communication is categorized into four primary types [20]:

- Device relaying with operator-controlled link establishment: A device in a remote area or far away from the eNB can communicate with an eNB using another device as a relay. In this communication scenario, an eNB can corroborate the relaying devices and thus preserve the privacy as well as frequency allocation of the devices.

- Direct D2D communication with operator-controlled link establishment: A network assisted link can communicate with each other bypassing the eNB. The eNB controls the connections, frequency allocation and pecuniary communication between devices.

- Device relaying with device-controlled link establishment: An eNB has no control over the device communication. Devices communicate with each other by using relays between them.

- Direct D2D communication with device-controlled link establishment: An eNB has no control over the device communication. Communication between devices is managed by the devices themselves.

Based on spectrum utilization, we can categorize D2D communication underlaying cellular networks into two primary categories as listed below [21].

1. Inband D2D communication/LTE Direct: D2D communication occurs in a cellular-licensed spectrum in a dedicated mode (overlay mode) or shared mode (underlay mode).

2. Outband D2D communication: D2D communication occurs in an unlicensed spectrum endorsed by other wireless technologies such as WiFi or Bluetooth.

The main advantages of integrating D2D communication in the traditional cellular networks are listed as follows: First, D2D communication reduces mobile traffic to the eNB, which results in a higher cellular network capacity. Next, D2D communication facilitates the reutilization of the available cellular spectrum, thus enabling maximum utilization of the available spectrum. Finally, D2D communication reduces end-to-end delay as it allows direct communication between nearby devices.

In the proposed scheme, we considered the aggregate interference caused by multiple D2D pairs. This affects the quality of the traditional cellular networks since the cellular users and D2D pairs shared the same resources. In addition, there are various technical challenges with the introduction of the D2D communication into the traditional cellular network such as time synchronization, frequency synchronization and peer discovery between the users. Consider an uplink cellular network scenario where the D2D pairs are reusing the uplink cellular links: This generates two main interferences, interference to the D2D receiver caused by the signal transmitted from the eNB to the cellular users, and interference to eNB caused by the signal transmitted by the D2D transmitter to D2D receiver. The co-channel interference caused by the D2D users to cellular users and vice versa deteriorates the system QoS and spectral efficiency of the overall network. Therefore, co-channel interference is considered the primary challenge in the underlay inband D2D communication system. To mitigate these interferences generated due to D2D communications, the FFR technique is primarily used by allotting specific spectrum resources to the users. The FFR scheme facilitates the reuse of the 
same frequency in cellular networks to encourage the concurrent communication demands. This can improve the system spectral efficiency and channel quality [22,23]. The FFR scheme utilizes the entire spectrum in a cell and reduces the co-channel interference within the cell as well as among the cells. Moreover, this interference can be avoided by introducing a complex resource allocation and power control method. The main drawback of using these methods is that it increases the computational overhead of the eNB. As a result, the performance of the traditional cellular users is deteriorated.

\section{Network Model}

In this paper, we consider an uplink multicell cellular network as shown in Figure 1. The reason for considering the hexagonal shape cell is to mitigate the propagation loss caused by the different terrains. Moreover, the hexagonal cellular geometry facilitates the analysis of cellular networks. In Figure 1, the cell area is divided into two regions namely inner and outer cell regions with cell radius $r$ and $R$, respectively. In each hexagonal cell, the base station is placed at the cell center. Both regions are sectored into three sub-regions using $120^{\circ}$ directional antennas. In this network, the reuse of the channel resources occurs in two scenarios: First, the D2D pairs in the inner cell region reuse the channel resources of the cellular users in the outer cell region. Second, the D2D pairs in the outer cell region reuse the channel resources of the cellular users in the inner cell region of the corresponding sectored region. The D2D pair located in a certain cell region can only reuse only one cellular resource of its corresponding cell region. Reusing the cellular resource by more than one D2D pair is strictly prohibited.

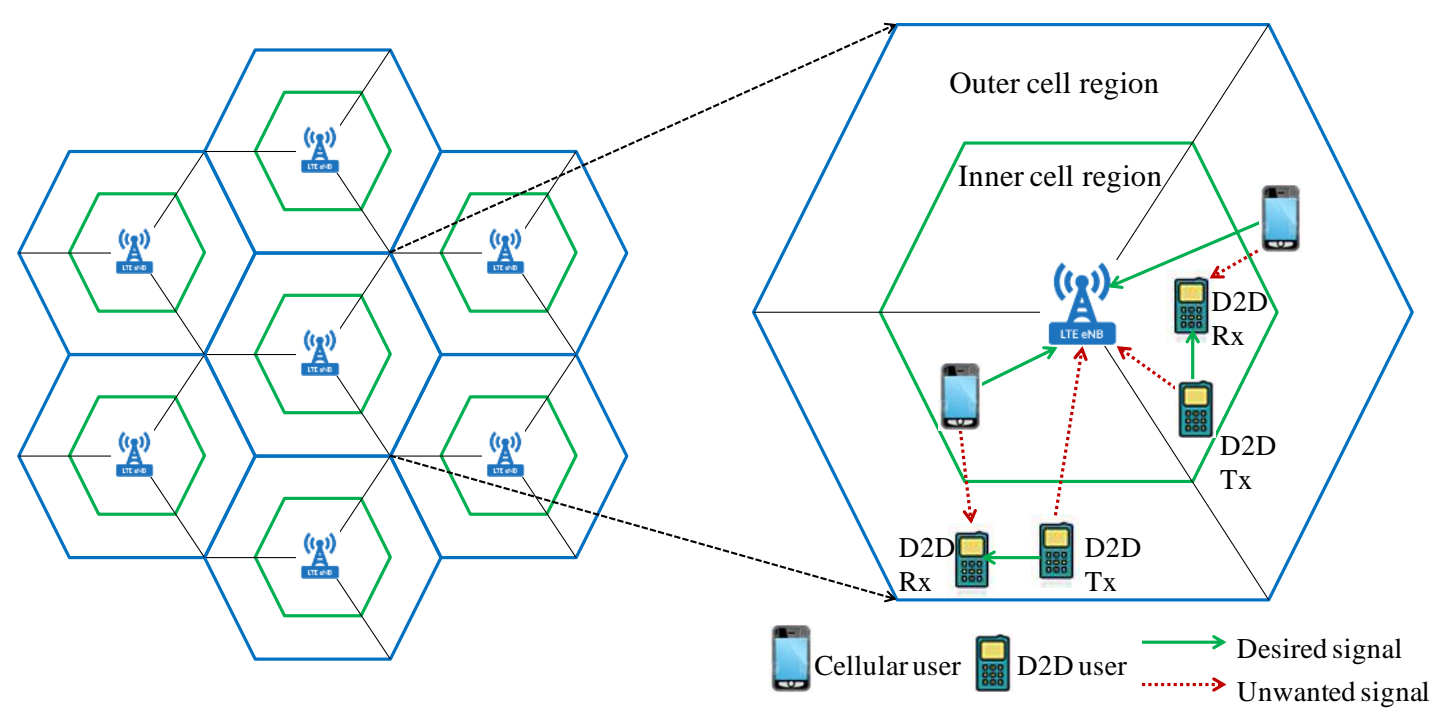

Figure 1. Device-to-device (D2D) communication network model.

Consider a cell with $M$ cellular users and $N$ D2D users uniformly distributed in a cell. We define the sets of cellular users and D2D users as $C=(1,2, \ldots, M)$ and $D=(1,2, \ldots, N)$, respectively. Similarly, the users are categorized as $C_{i n}, C_{\text {out }}, D_{\text {in }}$ and $D_{\text {out }}$, where $C_{\text {in }}$ and $C_{\text {out }}$ are the cellular users belonging to inner and outer cell regions, respectively, and $D_{\text {in }}$ and $D_{\text {out }}$ are the D2D users belongs to cell inner and outer region respectively. In this paper, we consider the distance between the D2D pair and the cellular user whose channel resource is to be reused by the D2D pair, in which a maximum separable distance is to be maintained to avoid interference from the cellular user to the device receiver. The partitioned frequency band structure based on the power level is shown in Figure 2a. In Figure 2a, initially the whole frequency band is partitioned into two parts, the inner cell frequency and the outer cell frequency. The non-orthogonal resource sharing between cellular users and D2D pairs is shown in Figure $2 \mathrm{~b}$. Further, both frequency bands are sub-divided into three corresponding bands as, $F_{I, 0}, F_{I, 1}, F_{I, 2}, F_{O, 0}, F_{O, 1}$ and $F_{O, 2}$, where $F_{I, 0}, F_{I, 1}$ and $F_{I, 2}$ are the sub-bands of the inner cell frequency 
$F_{I}$ and, $F_{O, 0}, F_{O, 1}$ and $F_{O, 2}$ are the sub parts of the outer cell frequency $F_{O}$. In our system model, we used the subparts of both frequencies to meet the design trade-off between spectrum utilization and interference mitigation in the cellular network.

$$
F=F_{I}+F_{O}=\left(F_{I, 0}+F_{I, 1}+F_{I, 2}\right)+\left(F_{O, 0}+F_{O, 1}+F_{O, 2}\right),
$$

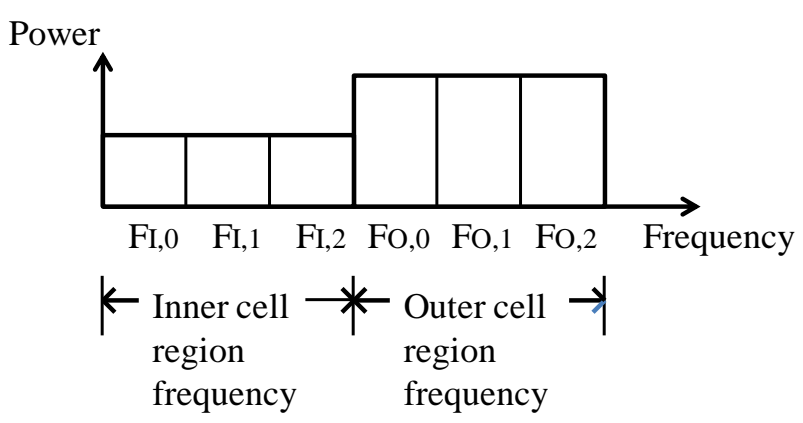

(a)

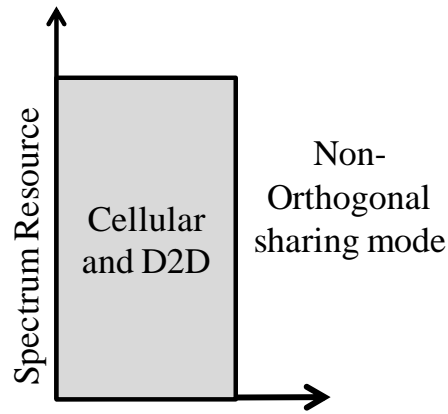

(b)

Figure 2. (a) Spectrum partitioning and (b) non-orthogonal spectrum resource sharing.

The location of the uniformly distributed cellular devices is expressed in terms of polar coordinates with an angle of $\theta_{C}$ and a distance $l_{c}$ from the eNB. Therefore, the probability density functions of the cellular user (in polar coordinates) in the inner and outer cell regions are expressed as follows [24]:

For the inner cell cellular users

$$
f\left(l_{C}\right)=\frac{2\left(l_{C}-l_{S}\right)}{\left(R-l_{S}\right)^{2}}, \forall l_{S} \leq l_{C} \leq R,
$$

For the outer cell cellular users

$$
\begin{gathered}
f\left(l_{c}\right)=\frac{2\left(l_{c}-l_{s}\right)}{\left(r-l_{s}\right)^{2}}, \forall l_{s} \leq l_{c} \leq r, \\
f\left(\theta_{c}\right)=\frac{1}{\left(\frac{2 \pi}{3}\right)}, \forall 0 \leq \theta_{c} \leq 2 \pi,
\end{gathered}
$$

where $l_{S}$ and $l_{S}$ are the shortest distance between the outer and the inner cell cellular users from the eNB, respectively; $R$ is the radius of the outer cell, and $r$ is the radius of the inner cell. The comprehensive functional flow of the proposed scheme is presented in Algorithm 1. Since the users are assisted by directional antennas, the available resources are uniformly allocated to all the users.

Moreover, the resource allocation scheme proposed in this paper is performed by considering different frequency sub-bands of the network. To allocate resources properly, we assumed that a D2D pair located in $F_{I, 0}$ sub-band cannot reuse the resources of same sub-band but can reuse the resources of $F_{O, 0}$. In addition, the distance between the cellular user located in $F_{O, 0}$ sub-band and D2D pair located in $F_{I, 0}$ sub-band should maintain. This results in the least interference between the cellular user and D2D pair. 


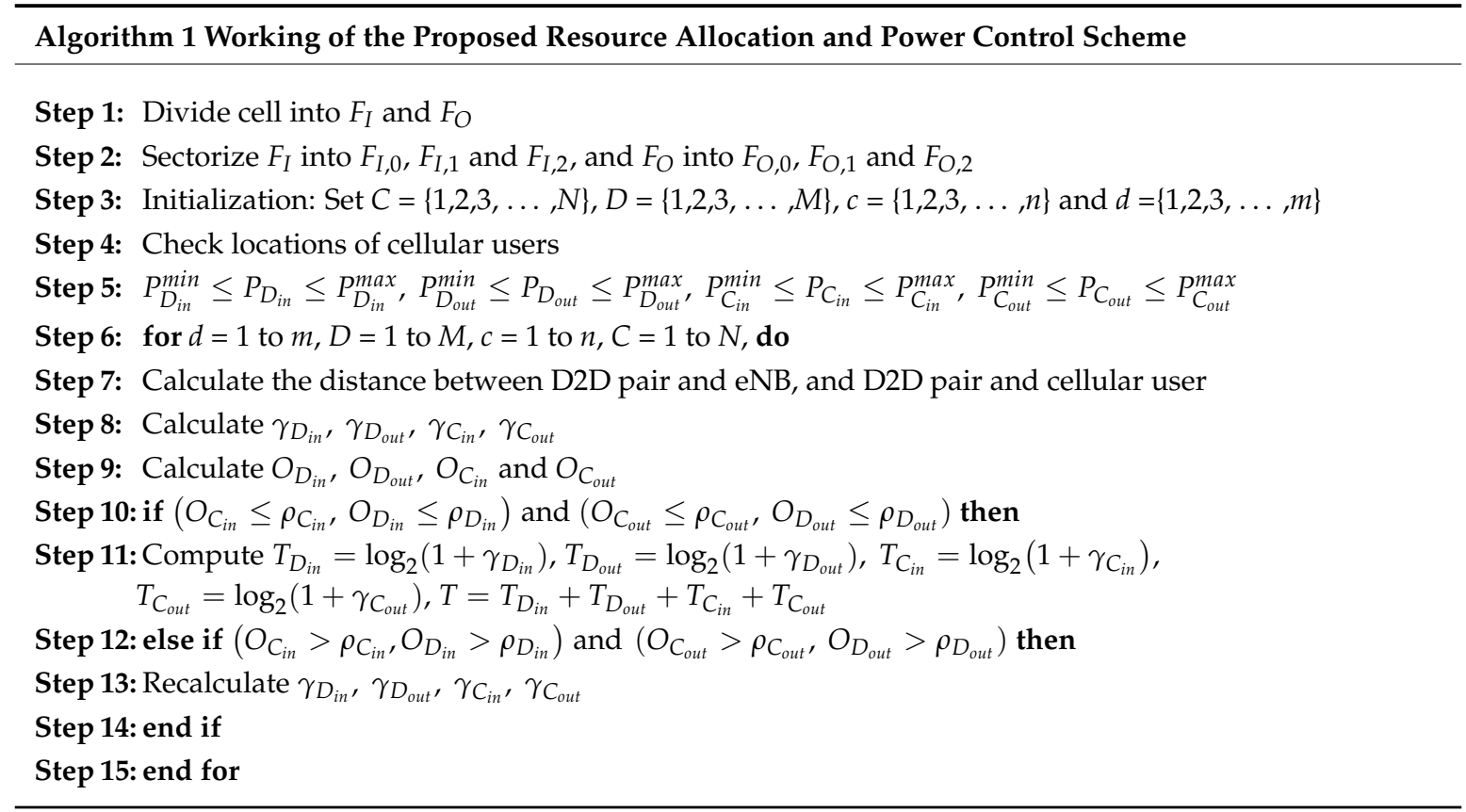

\section{Problem Formulation}

\subsection{Interference Analysis}

The received power in the communication system is defined as the product of the transmitted power and the channel coefficient i.e.,

$$
\begin{gathered}
P_{R}=P_{T} \times \mathrm{G}, \\
\mathrm{G}=P_{\mathrm{g}} \times \phi^{-1} \times \delta,
\end{gathered}
$$

where $P_{R}$ is the received power, $P_{T}$ is the transmitted power, $G$ is the channel coefficients, $P_{g}$ is the power gain, $\phi$ is the path loss and $\delta$ is the shadowing coefficient.

To maintain reliable information transmission after integrating D2D communications in the cellular networks, the SINR requirement is set up to a minimum level for the D2D communication. We assumed that in the inner cell region, D2D transmitter $b$ communicates with receiver $b^{\prime}$ by reusing resource of the cellular user $j$ as shown in section S-1 of Figure 3. Similarly, in the outer cell region, D2D transmitter $e$ communicates with receiver $e^{\prime}$ by reusing resource of the cellular user $a$, as shown in section S-2 of Figure 3. The eNB is denoted as $B$ for analysis purpose.

Considering the interference scenario presented in Figure 4, we can express the SINR of cellular users and the D2D users of both the inner and outer cell regions as follows:

- For the inner cell user: The received SINR for the inner cell cellular user and the D2D pair is given by

$$
\begin{gathered}
\gamma_{C_{i n}}=\frac{P_{a} A_{a, B}^{-\alpha}\left|G_{a, B}\right|^{2}}{P_{N}+P_{e} A_{e, B}^{-\alpha}\left|G_{e, B}\right|^{2}} \geq \gamma_{t}, \forall a \in C_{\text {in }}, e \in D_{\text {out }}, \\
\gamma_{D_{\text {in }}=}=\frac{P_{b} A_{b, b^{\prime}}^{-\alpha}\left|G_{b, b^{\prime}}\right|^{2}}{P_{N}+P_{j} A_{j, b^{\prime}}^{-\alpha}\left|G_{j, b^{\prime}}\right|^{2}+I_{b^{\prime}}+I_{\text {ng }}} \geq \gamma_{t}, \forall b, b^{\prime} \in D_{\text {in }}, j \in C_{\text {out }},
\end{gathered}
$$

respectively, where $I_{b^{\prime}}$ is the interference to D2D receiver $b^{\prime}$ in the inner cell region and can be expressed as follows:

$$
I_{b^{\prime}}=\sum_{m=1}^{2} P_{m} A_{m, b^{\prime}}^{-\alpha}\left|G_{m, b^{\prime}}\right|^{2}, \forall m, b^{\prime} \in D_{i n}
$$


The interference from the neighboring cells $I_{n g}$ can be denoted as

$$
I_{n g}=\sum_{i=1}^{6} I_{i}
$$

- $\quad$ For the outer cell user: The received SINR for the outer cell cellular user and the D2D pair is given by

$$
\begin{gathered}
\gamma_{C_{\text {out }}}=\frac{P_{j} A_{j, B}^{-\alpha}\left|G_{j, B}\right|^{2}}{P_{N}+P_{b} A_{b, B}^{-\alpha}\left|G_{b, B}\right|^{2}} \geq \gamma_{t}, \forall b \in D_{\text {in }}, j \in C_{\text {out }}, \\
\gamma_{D_{\text {out }}}=\frac{P_{e} A_{e, e^{\prime}}^{-\alpha}\left|G_{e, e^{\prime}}\right|^{2}}{P_{N}+P_{a} A_{a, e^{\prime}}^{-\alpha}\left|G_{a, e^{\prime}}\right|^{2}+I_{e^{\prime}}+I_{n}} \geq \gamma_{t}, \forall e, e^{\prime} \in D_{\text {out }}, a \in C_{\text {in }},
\end{gathered}
$$

respectively, where $I_{e^{\prime}}$ is the interference to user D2D receiver $e^{\prime}$ and can be listed as

$$
\begin{gathered}
I_{e^{\prime}}=\sum_{n=1}^{2} P_{n} A_{n, e^{\prime}}^{-\alpha}\left|G_{n, e^{\prime}}\right|^{2}, \forall e^{\prime}, n \in D_{\text {out }}, \\
I_{n g}=\sum_{q=1}^{6} I_{q,}
\end{gathered}
$$

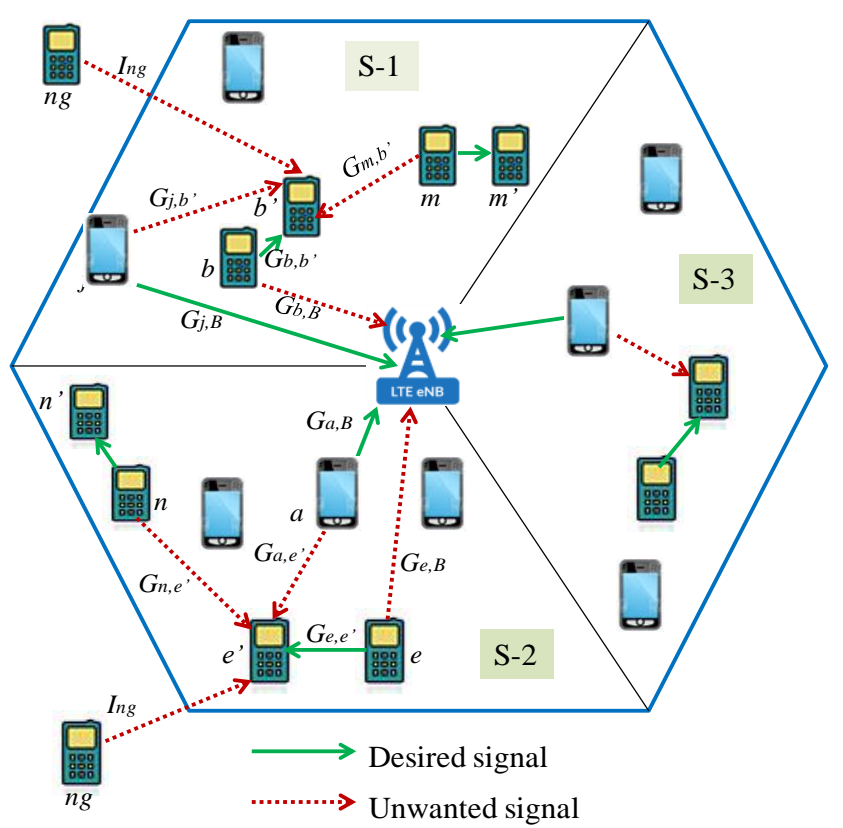

\begin{tabular}{l}
\multicolumn{1}{c}{ Notations } \\
$\boldsymbol{b}, \boldsymbol{m}=$ inner cell D2D \\
transmitters \\
$\boldsymbol{b}^{\prime}, \boldsymbol{m}$ '= inner cell D2D \\
receivers \\
$\boldsymbol{a}=$ inner cell cellular \\
user \\
$\boldsymbol{j}=$ outer cell cellular \\
user \\
$\boldsymbol{e}, \boldsymbol{n}=$ outer cell D2D \\
transmitters \\
$\boldsymbol{e}, \boldsymbol{n}^{\prime}=$ outer cell D2D \\
receivers \\
$\boldsymbol{n} \boldsymbol{g}=$ neighboring users
\end{tabular}

Figure 3. Single uplink cellular resource reused by one D2D pair.

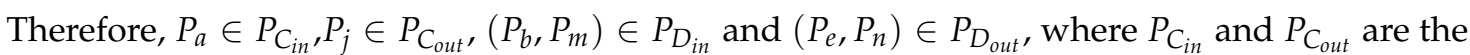
transmit power of the cellular user in the inner and outer cell regions, respectively, $P_{D_{\text {in }}}$ and $P_{D_{\text {out }}}$ are the transmit power of the D2D user in the inner and outer cell regions, respectively. Similarly, $A_{a, B}$ and $A_{e, B}$ are the distance between the inner cell cellular user $a$ and $B$, and distance between the outer cell D2D transmitter $e$ and $B . A_{b, b^{\prime}}, A_{j, b^{\prime}}$ and $A_{m, b^{\prime}}$ distance between the inner cell D2D transmitter $b$ and receiver $b^{\prime}$, distance between the outer cell cellular user $j$ and inner cell D2D receiver $b^{\prime}$, and the distance between the co-channel D2D transmitter $m$ and receiver $b^{\prime} . A_{j, B}, A_{b, B}$ are the distance between the outer cell cellular user $j$ and $B$, and distance between the inner cell D2D transmitter $b$ and $B . A_{e, e^{\prime}}, A_{a, e^{\prime}}$ and $A_{n, e^{\prime}}$ are the distance between outer the cell D2D transmitter $e$ and receiver $e^{\prime}$, distance between the 
co-channel D2D transmitter $n$ and $e^{\prime}$. Moreover, $G_{a, B}$ and $G_{e, B}$ are the channel coefficient between the inner cell cellular user $a$ and $B$, and channel coefficient between the outer cell D2D transmitter $e$ and $B . G_{b, b^{\prime}}, G_{j, b^{\prime}}$ and $G_{m, b^{\prime}}$ are the channel coefficient between the inner cell D2D transmitter $b$ and receiver $b^{\prime}$, channel coefficient between the outer cell cellular user $j$ and inner cell D2D receiver $b^{\prime}$, and channel coefficient between the co-channel D2D transmitter $m$ and $b^{\prime} . G_{j, B}$ and $G_{b, B}$ are the channel coefficient between the outer cell cellular user $j$ and $B$, and channel coefficient between the inner cell D2D transmitter $b$ and B. $G_{e, e^{\prime}}, G_{a, e^{\prime}}$ and $G_{n, e^{\prime}}$ are the channel coefficient between the outer cell D2D transmitter $e$ and receiver $e^{\prime}$, channel coefficient between the inner cell cellular user $a$ and outer cell D2D receiver $e^{\prime}$, and channel coefficient between the co-channel D2D transmitter $n$ and receiver $e^{\prime}$. $P_{N}$ is the noise power, $\alpha$ is the path-loss exponent, and $I_{n g}$ is the interference from the neighboring cells to the D2D receivers.

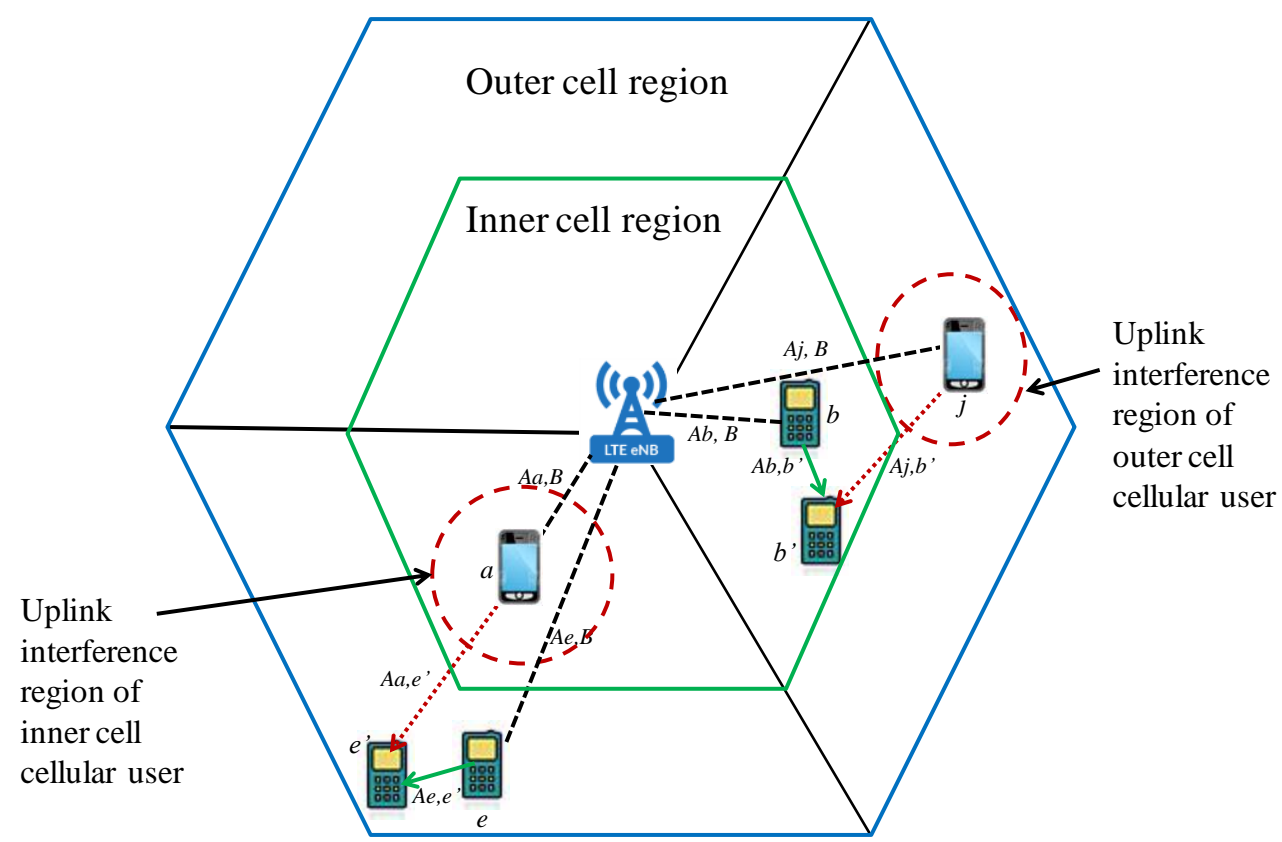

Figure 4. Uplink interference regions of cellular users.

In order to guarantee the QoS of the D2D users, we defined an uplink interference region of the cellular users as shown in Figure 4. Within this region, interference from cellular user to D2D receiver is very severe, hence D2D communication is not allowed in this region [25]. For interference region analysis, we consider a scenario where a communication system attains a minimum allowable SINR. From Equations (7)-(12), we defined the uplink interference region $\left(U_{C_{i n}}\right)$ as listed below.

$$
\left.\begin{array}{l}
\begin{array}{l}
P_{a} A_{a, B}^{-\alpha}\left|G_{a, B}\right|^{2} \\
P_{e} A_{e, B}^{-\alpha}\left|G_{e, B}\right|^{2}
\end{array}=\gamma_{t}, \\
\frac{P_{a}}{P_{e}}=\gamma_{t} \frac{A_{e, B}^{-\alpha}\left|G_{e, B}\right|^{2}}{A_{a, B}^{-\alpha}\left|G_{a, B}\right|^{2}}
\end{array}\right\},
$$


From Equations (15) and (16), we have

$$
\left.\begin{array}{c}
A_{t}^{-\alpha} \frac{A_{a, e^{\prime}}\left|G_{a, e^{\prime}}\right|^{2}}{A_{e, e^{\prime}}^{-\alpha}\left|G_{e, e^{\prime}}\right|^{2}}=\frac{A_{a, B}^{-\alpha}\left|G_{a, B}\right|^{2}}{\gamma_{t}\left(A_{e, B}^{-\alpha}\left|G_{e, B}\right|^{2}\right)^{\prime}} \\
\gamma_{t}^{2}=\frac{\left.A_{a, B}^{-\alpha} G_{a, B}\right|^{2}}{A_{e, B}^{-\alpha}\left|G_{e, B}\right|^{2}} \times \frac{A_{e, e^{\prime}}^{-\alpha}\left|G_{e, e^{\prime}}\right|^{2}}{A_{a, e^{\prime}}^{-\alpha}\left|G_{a, e^{\prime}}\right|^{2}}, \\
=\left(\frac{A_{a, B}}{A_{e, B}} \cdot \frac{A_{e, e^{\prime}}}{A_{a, e^{\prime}}}\right)^{-\alpha} \times\left(\frac{\left|G_{a, B}\right|}{\left|G_{e, B}\right|} \cdot \frac{\left|G_{e, e^{\prime}}\right|}{\left|G_{a, e^{\prime}}\right|}\right),{ }^{2}
\end{array}\right\},
$$

In Equation (17), we assume $\left(\frac{\left|G_{a, B}\right|}{\left|G_{e, B}\right|} \cdot \frac{\left|G_{e, e^{\prime}}\right|}{\left|G_{a, e^{\prime}}\right|}\right)=\gamma_{t}=$ constant value, then

$$
A_{a, e^{\prime}}=U_{C_{i n}} \geq\left(\frac{A_{a, B} A_{e, e^{\prime}}}{A_{e, B}}\right)
$$

We can see from Equation (18) that to guarantee the QoS of the D2D communication in outer cell region by reusing inner cell cellular resource, the distance between inner cell cellular user and outer cell D2D receiver should be large.

Similarly, from Equations (8) and (11) we defined the uplink interference region $\left(U_{C_{\text {out }}}\right)$ as listed below.

$$
\begin{aligned}
& \frac{P_{b} A_{b, b^{\prime}}^{-\alpha}\left|G_{b, b^{\prime}}\right|^{2}}{P_{j} A_{j, b^{\prime}}^{-\alpha}\left|G_{j, b^{\prime}}\right|^{2}}=\gamma_{t}, \\
& \left.\frac{P_{b}}{P_{j}}=\gamma_{t} \frac{A_{j, b^{\prime}}^{-\alpha}\left|G_{j, b^{\prime}}\right|^{2}}{A_{b, b^{\prime}}^{-\alpha}\left|G_{b, b^{\prime}}\right|^{2}},\right\}, \\
& \left.\begin{array}{l}
\frac{P_{j} A_{j, B}^{-\alpha}\left|G_{j, B}\right|^{2}}{P_{b} A_{b, B}^{-\alpha}\left|G_{b, B}\right|^{2}}=\gamma_{t}, \\
\frac{P_{j}}{P_{b}}=\gamma_{t} \frac{A_{b, B}^{-\alpha}\left|G_{b, B}\right|^{2}}{A_{j, B}^{-\alpha}\left|G_{j, B}\right|^{2}}
\end{array}\right\},
\end{aligned}
$$

From Equations (19) and (20), we have,

$$
\left.\begin{array}{c}
\gamma_{t} \frac{A_{b, B}^{-\alpha}\left|G_{b, B}\right|^{2}}{A_{j, B}^{-\alpha}\left|G_{j, B}\right|^{2}}=\frac{A_{b, b^{\prime}}^{-\alpha}\left|G_{b, b^{\prime}}\right|^{2}}{\gamma_{t}\left(A_{j, b^{\prime}}^{-\alpha}\left|G_{j, b^{\prime}}\right|^{2}\right)^{\prime}}, \\
\gamma_{t}^{2}=\frac{A_{b, b^{\prime}}^{-\alpha}\left|G_{b, b^{\prime}}\right|^{2}}{A_{j, b^{\prime}}\left|G_{j, b^{\prime}}\right|^{2}} \times \frac{A_{j, B}^{-\alpha}\left|G_{j, B}\right|^{2}}{A_{b, B}^{-\alpha}\left|G_{b, B}\right|^{2}}, \\
=\left(\frac{A_{b, b_{1}}}{A_{j, b}} \cdot \frac{A_{j, B}}{A_{b, B}}\right)^{-\alpha} \times\left(\frac{\left|G_{b, b^{\prime}}\right|}{\left|G_{j, b^{\prime}}\right|} \cdot \frac{\left|G_{j, B}\right|}{\left|G_{b, B}\right|}\right)^{2},
\end{array}\right\},
$$

In Equation (17), we assume $\left(\frac{\left|G_{b, b^{\prime}}\right|}{\left|G_{j, b^{\prime}}\right|} \cdot \frac{\left|G_{j, B}\right|}{\left|G_{b, B}\right|}\right)=\gamma_{t}=$ constant value, then

$$
A_{j, b^{\prime}}=U_{C_{o u t}} \geq\left(\frac{A_{b, b} A_{j, B}}{A_{b, B}}\right),
$$

We can see from Equation (22) that to guarantee the QoS of the D2D communication in inner cell region by reusing outer cell cellular resource, the distance between outer cell cellular user and inner cell D2D receiver should be large. Therefore, a larger distance between the users can mitigate uplink interference from the cellular user to D2D communication. In this way, we can improve D2D communication throughput and overall system throughput.

\subsection{Outage Probability Analysis}

Outage probability is important in analyzing the performance of wireless communication systems. In this paper, we analyze the outage probabilities of the D2D communications and cellular networks in 
a multicell system when the uplink cellular links are shared by the D2D pairs. The outage probability is stated as the probability that the spontaneous SINR drops below a predefined threshold SINR. Therefore, the corresponding outage probability of a cellular user and D2D pair in both cell regions in terms of their SINRs are illustrated as below.

- For the inner cell user: The outage probability for the cellular user in the inner cell region, in terms of the received SINR is given as

$$
\begin{aligned}
& \left.\begin{array}{c}
O_{C_{\text {in }}}=1-\frac{1}{1+\frac{\left(P_{\mathrm{e}} A_{\mathrm{e}, B}^{-\alpha}\right) \gamma_{t}}{P_{a} A_{a, B}^{-\alpha}}} \exp \left(-\frac{\gamma_{t} P_{N}}{P_{a}}\right) \leq \rho_{C_{i n},} \\
=1-\frac{P_{a} A_{a, B}^{-\alpha}}{P_{a} A_{a, B}^{-\alpha}+\left(P_{\mathrm{e}} A_{\mathrm{e}, B}^{-\alpha}\right) \gamma_{t}} \mathrm{e}^{\left(-\frac{P_{N} \gamma^{T h}}{P_{a}}\right)} \leq \rho_{C_{i n},}
\end{array}\right\}, \forall a \in C_{i n}, e \in D_{\text {out }}, \\
& \left.\begin{array}{c}
O_{D_{\text {in }}}=1-\frac{1}{1+\frac{\left(P_{j} A_{j, b^{\prime}}^{-\alpha}+I_{b^{\prime}}+I_{n}\right) \gamma_{t}}{P_{b} A_{b, b^{\prime}}^{-\alpha}}} \exp \left(-\frac{\gamma_{t} P_{N}}{P_{b}}\right) \leq \rho_{D_{\text {in }}} \\
=1-\frac{P_{b} A_{b, b^{\prime}}^{-\alpha}}{P_{b} A_{b, b^{\prime}}^{-\alpha}+\left(P_{j} A_{j, b^{\prime}}^{-\alpha}+I_{b^{\prime}}+I_{n}\right) \gamma_{t}} \mathrm{e}^{\left(-\frac{P_{N} \gamma^{T h}}{P_{b}}\right)} \leq \rho_{D_{\text {in }},}
\end{array}\right\}, \forall b, b^{\prime} \in D_{\text {in }}, j \in C_{\text {out }},
\end{aligned}
$$

where $\gamma_{t}, \rho_{C_{i n}}$ and $\rho_{D_{\text {in }}}$ are the received SINR threshold, predefined outage probability requirement of the cellular user and D2D user, respectively.

Similarly, the outage probability for the inner cell D2D pair in terms of received SINR is given by:

- For the outer cell user: The outage probability for the cellular user and the D2D pair in the outer cell region in terms of their SINRs is expressed as

$$
\begin{aligned}
& \left.\begin{array}{c}
O_{C_{\text {out }}}=1-\frac{1}{1+\frac{\left(P_{b} A_{b, B}^{-\alpha}\right) \gamma_{T}}{P_{j} A_{j, B}^{-\alpha}}} \exp \left(-\frac{\gamma_{T} P_{N}}{P_{j}}\right) \leq \rho_{C_{\text {out }},} \\
=1-\frac{P_{j} A_{j, B}^{-\alpha}}{P_{j} A_{j, B}^{-\alpha}+\left(P_{b} A_{b, B}^{-\alpha}\right) \gamma_{T}} \mathrm{e}^{\left(-\frac{P_{N} \gamma^{T h}}{P_{j}}\right)} \leq \rho_{C_{\text {out }},}
\end{array}\right\}, \forall b \in D_{\text {in }}, j \in C_{\text {out }}, \\
& \left.\begin{array}{c}
O_{D_{\text {out }}}=1-\frac{1}{1+\frac{\left(P_{a} A_{a, e^{\prime}}^{-\alpha} I_{e^{\prime}}+I_{n}\right) \gamma_{T}}{P_{e} A_{e, e^{\prime}}^{-\alpha}}} \exp \left(-\frac{\gamma_{T} P_{N}}{P_{e}}\right) \leq \rho_{D_{\text {out }},} \\
=1-\frac{P_{e} A_{e, e^{\prime}}^{-\alpha}}{P_{e} A_{e, e^{\prime}}^{-\alpha}+\left(P_{a} A_{a, e^{\prime}}^{-\alpha}+I_{e^{\prime}}+I_{n}\right) \gamma_{T}} \mathrm{e}^{\left(-\frac{P_{N} \gamma^{T h}}{P_{e}}\right)} \leq \rho_{D_{\text {out }},}
\end{array}\right\}, \forall e, e^{\prime} \in D_{\text {out }}, a \in C_{\text {in }},
\end{aligned}
$$

where $\gamma_{T}, \rho_{C_{\text {out }}}$ and $\rho_{D_{\text {out }}}$ are the received SINR threshold, predefined outage probability requirement of the cellular user and D2D user, respectively.

\section{System Throughput Optimization}

The throughput is defined as

$$
T=\log _{2}(1+\gamma)
$$

where $\gamma$ denotes the user's SINR. Combining the SINR Equations (7)-(22) of different users in both cell regions, we can then formulate the overall system throughput optimization problem as follows:

$$
\max \sum_{C=1}^{N} \sum_{D=1}^{M}\left[T_{C_{\text {in }}}+T_{C_{\text {out }}}+T_{D_{\text {in }}}+T_{D_{\text {out }}}\right]
$$

where

$$
T_{C_{\text {in }}}=\log _{2}\left(1+\gamma_{C_{\text {in }}}\right), T_{C_{\text {out }}}=\log _{2}\left(1+\gamma_{C_{\text {out }}}\right)
$$




$$
T_{D_{\text {in }}}=\log _{2}\left(1+\gamma_{D_{\text {in }}}\right), T_{D_{\text {out }}}=\log _{2}\left(1+\gamma_{D_{\text {out }}}\right)
$$

subject to

$$
\begin{gathered}
\gamma_{C} \geq \gamma_{C}^{\text {min }}, \gamma_{D} \geq \gamma_{D}^{\text {min }} \\
P_{C}^{\text {min }} \leq P_{C} \leq P_{C}^{\text {max }}, P_{D}^{\text {min }} \leq P_{D} \leq P_{D}^{\max }
\end{gathered}
$$

where $\gamma_{C}^{\text {min }}$ and $\gamma_{D}^{\min }$ are the minimum achievable SINRs of the cellular user and D2D user respectively. $P_{C}^{\min }$ and $P_{C}^{\max }$ are the minimum and maximum power range for the cellular user, and $P_{D}^{\min }$ and $P_{D}^{\max }$ are the minimum and maximum power ranges of the D2D user. The optimization problem in Equation (28) gives the throughput maximization by considering throughput of both the cellular users and D2D pairs. Constraints in Equation (31) stand for SINR of both the cellular users and D2D pairs should be respectively greater than or equal to the minimum required SINR. Constraints listed in Equation (32) represent that the power level of all users should range from its predefined maximum and minimum levels. In a multicell D2D communication underlaying an uplink cellular network, the resource pairing criteria between cellular users and D2D pairs directly impact the transmit power of each user. Therefore, to specify a definite power range for each user is a key criterion for our proposed technique.

Proposition: The optimal solution of the optimization problem in Equation (28) must be the global-optimal solution of the proposed optimization problem.

Proof: As a matter of fact, when a D2D pair underlaying uplink cellular network reused the cellular resource, the power control mechanism is directly related with the throughput maximization problem. The upper and lower bound transmit power of both the users in cell inner and outer regions can be derived as:

$$
\begin{aligned}
& P_{a}=\frac{\gamma C_{i n}\left(P_{N}+P_{e} A_{e, B}^{-\alpha}\left|G_{e, B}\right|^{2}\right)}{A_{a, B}^{-\alpha}\left|G_{a, B}\right|^{2}}, P_{b}=\frac{\gamma_{D_{i n}}\left(P_{N}+P_{j} A_{j, b^{\prime}}^{-\alpha}\left|G_{j, b^{\prime}}\right|^{2}+I_{b^{\prime}}+I_{n g}\right)}{A_{b, b^{\prime}}^{-\alpha}\left|G_{b, b^{\prime}}\right|^{2}}, \quad \forall a \in C_{i n}, b \in D_{i n}, \\
& \left.P_{j}=\frac{\gamma_{C_{\text {out }}}\left(P_{N}+P_{b} A_{b, B}^{-\alpha}\left|G_{b, B}\right|^{2}\right)}{A_{j, B}^{-\alpha}\left|G_{j, B}\right|^{2}}, P_{e}=\frac{\gamma_{D_{\text {out }}}\left(P_{N}+P_{a} A_{a, e^{\prime}}\left|G_{a, e^{\prime}}\right|^{2}+I_{e^{\prime}}+I_{n}\right)}{A_{e, e^{\prime}}^{-\alpha}\left|G_{e, e^{\prime}}\right|^{2}},\right\}^{\prime} j \in C_{\text {out }}, e \in D_{\text {out }}
\end{aligned}
$$

respectively, where $C_{\text {in }}, C_{\text {out }} \in C$ and $D_{\text {in }}, D_{\text {out }} \in D$.

According to Equation (31), if $\gamma_{C}^{\min }>\gamma_{C}$ and $\gamma_{D}^{\min }>\gamma_{D}$, then the desired signal cannot be detected. This directly impacts the performance of the network. Consequently, to gratify the minimum SINR, it is necessary to control transmit power of both the users. Hence, $P_{C}^{\min } \leq P_{C} \leq P_{C}^{\max }, P_{D}^{\min } \leq$ $P_{D} \leq P_{D}^{\max }$ should be satisfied. This ensures that the transmit power of both the users does not exceed the maximum level. This completes the proof.

\section{Channel Model}

The fundamental principle for an eNB to adopt a device operating in either the cellular or D2D mode is determined by the location of the device transmitter and receiver versus the eNB. Therefore, the path loss (PL) measurement is notably considered as the benchmark for determining the device's operating mode. The path losses of different communication links in this paper are adopted using micro-urban models specified by the International Telecommunication Union Radio-Communication Sector (ITU-R) reports. The path loss models from the base station to cellular users and D2D pairs are calculated as follows [26]:

$$
\left.\begin{array}{c}
P L_{C}=36.7 \log _{10}(L)+26 \log _{10}\left(\frac{F_{c}}{5}\right)+40.9, \\
P L_{D}=40 \log _{10}\left(\frac{L}{1000}\right)+30 \log _{10}\left(\frac{F_{c}}{1000}\right)+49,
\end{array}\right\},
$$

where $L$ is the distance between the transmitter and receiver in meters, and $F_{c}$ is the carrier frequency in GHz. 


\section{Computational Complexity and Use Case Scenario of the Proposed Scheme}

\subsection{Computational Complexity Analysis}

To improve system throughput by minimizing interference, Algorithm 1 allows the limited communication by defining upper and lower bounds transmit power. In Algorithm 1, all available channels for cellular users and D2D users are evaluated. This results in a complexity of $O(M N)$. It is important to note that the complexity of the analysis increases exponentially with the number of users. Furthermore, our proposed scheme assumes that each D2D pair can only reuse single cellular resource at a time. Therefore, the complexity becomes $O\left(M^{2} N\right)$. The conditions $\left(O_{C_{i n}} \leq \rho_{C_{i n}}, O_{D_{i n}} \leq \rho_{D_{i n}}\right)$ and $\left(O_{C_{\text {out }}} \leq \rho_{C_{\text {out }}}, O_{D_{\text {out }}} \leq \rho_{D_{\text {out }}}\right)$ are the check points for the system outage level. These conditions control the number of iterations, hence reduces computational complexity of the system. Thus, our proposed resource allocation and power control scheme for D2D communications is a low complexity state-of-the-art solution, applicable for high data rate services.

\subsection{Use Case Scenario of the Proposed Scheme}

D2D communications provide various types of advantages over the conventional cellular networks. In this section, we provide some use case example scenarios of our proposed scheme. The basic concept of the proposed scheme is to allow more number of users in the network to jointly achieve higher system throughput and mitigate interference. In particular, some of the popular real-time use case scenarios are: public safety and traffic offloading. In the proposed scheme, we would like to analyze the use case scenario "traffic offloading" as an example. In this scenario, D2D users setup direct connections and triggered D2D communication by reusing available cellular resources without the control of eNB. Devices in proximity exhibit their preferences by analyzing SINR requirements. This helps to reduce the eNB from traffic burden and greatly maximizes the overall system throughput.

\section{Performance Evaluation}

Herein, to evaluate the performance of the proposed resource allocation and power control using the FFR scheme, we analyzed both the mathematical computation and simulation using the Monte Carlo Simulation. The proposed scheme considers full loaded cellular system that can support a high density of users. The number of cellular users in all sections of the cell is uniformly distributed and at least one D2D pair should be present in each cell section. We consider four different scenarios for sharing cellular resources with the D2D pairs. We compare the performances of our proposed scheme with the RRA scheme [2] and RA w/o cell sectorization scheme [16]. The above mentioned references and our proposed scheme have the same characteristics of the problem statement but with different system models. The methods considered core characteristics of the uplink cellular resources like channel bandwidth, interference scenarios, noise power, user distribution model, etc. The resource-sharing scenarios with different densities of both cellular users and D2D pairs are listed below:

1. When the D2D pairs are more than the cellular users (D2D $>C$ ) as given in Table 1, and the D2D transmitter $(\mathrm{Tx})$ and receiver $(\mathrm{Rx})$ are located in different cell sections: In this scenario, all the available cellular resources can be reused by the D2D pairs. This results in the maximum utilization of the spectrum, but the performance of the D2D communication may not be efficient, the number of available resources to be reused by the D2D pairs is less. As the D2D Tx and Rx are located in different sections of the cell, the D2D Rx will be interfered more by the nearby cellular users.

2. When the D2D pairs are more than the cellular users (D2D > C) as given in Table 1, and the D2D transmitter (Tx) and receiver ( $\mathrm{Rx}$ ) are located in same cell section: In this scenario, as both the D2D Tx and Rx are located in the same cell section, the D2D communication have less interfering devices to the D2D Rx. This scenario is more efficient than scenario 1. 
3. When the number of cellular users are more than the number of D2D Pairs $(C>D 2 D)$ as given in Table 2, and the D2D transmitter (Tx) and receiver (Rx) are located in different cell sections: Here, more cellular links will not be utilized by the D2D pairs as the number of sources is high but the consumers are few. However, when some of the available cellular links are disconnected or are in sleep mode, the D2D pair can have other options to choose the remaining active cellular links. This condition cannot apply to the first and second scenarios.

4. When the number of cellular users is more than the number of D2D Pairs (C > D2D) as given in Table 2, and the D2D transmitter (Tx) and receiver (Rx) are located in the same cell section: As the D2D Tx and Rx exist in the same cell section, the communication will have less interference.

Table 1. Scenario 1 and 2: D2D > C.

\begin{tabular}{cc}
\hline Cell Region & Number of Devices \\
\hline Cell inner region & D2D $=15, C=40$ \\
Cell outer region & D2D $=50, C=10$ \\
\hline
\end{tabular}

Table 2. Scenario 3 and 4: C > D2D.

\begin{tabular}{cc}
\hline Cell Region & Number of Devices \\
\hline Cell inner region & $\mathrm{D} 2 \mathrm{D}=15, \mathrm{C}=50$ \\
Cell outer region & $\mathrm{D} 2 \mathrm{D}=30, \mathrm{C}=30$ \\
\hline
\end{tabular}

\subsection{Simulation Parameters and Values}

This section provides the detailed simulation parameters and its values as given in Table 3 . Without loss of generality, we assumed that the transmitter and receiver of a D2D pair are placed in the same section of the cell and distance between them is short, i.e., 1-50 $\mathrm{m}$.

Table 3. Parameters used in simulation.

\begin{tabular}{cc}
\hline Parameter & Value \\
\hline Cell outline & 7-cells, hexagonal framework \\
evolved-NodeB (Enb) transmission power & $40-46 \mathrm{dBm}$ (inner cell) \\
eNB transmission power & $43-49 \mathrm{dBm}$ (outer cell) \\
D2D transmission power & $8-15 \mathrm{dBm}$ \\
Noise power & $-174 \mathrm{dBm} / \mathrm{Hz}$ \\
D2D pair distance & $1-50 \mathrm{~m}$ \\
Carrier frequency & $2 \mathrm{GHz}$ \\
Cell radius & $500 \mathrm{~m}$ \\
Uplink bandwidth & $5 \mathrm{MHz}$ \\
Path loss exponent & 4 \\
Antenna type & $3^{\circ}-120^{\circ}$ directional antennas \\
Monte-Carlo simulation runs & 15,000 \\
\hline
\end{tabular}

\subsection{Simulation Results and Discussions}

This section demonstrates the simulation results and shows the performance comparison. Figure $5 \mathrm{a}, \mathrm{b}$ show the average target SINR vs. cumulative distribution function (CDF) plot for both the D2D pairs. This Figure shows that the performance of the system with more D2D pairs than cellular users achieved closer to the average target SINR compared to the communication scenario with less D2D pairs. Our proposed scheme also benefit SINR of around $(\approx 3.5 \mathrm{~dB})$ at the outer cell D2D users compared to the existing schemes. This is because of the effective distribution of the SINR based on the distance between the D2D pairs and cellular users. From the Figure $5 \mathrm{a}$, it is seen that in all the methods, $60 \%$ of D2D users can set up communication with SINR from $-7 \mathrm{~dB}$ to $10 \mathrm{~dB}$. Similarly, it is clearly seen from Figure $5 \mathrm{~b}$ that in all the methods, $60 \%$ of D2D users can set up communication 
with SINR from $23 \mathrm{~dB}$ to $30 \mathrm{~dB}$. As expected, RRA scheme attains least SINR in comparison with our proposed scheme and RA w/o sectorization scheme.

Figure $6 \mathrm{a}, \mathrm{b}$ show the average target SINR vs. cumulative distribution function (CDF) plot for both the cellular users. The performance of the cellular users is affected little by the varying number of D2D pairs in both cell regions. Also, cellular users in inner cell region attain a gain of somewhat $(\approx 4 \mathrm{~dB})$ from the existing schemes. From Figure $6 \mathrm{a}$, it is seen that in all the communication scenarios, $60 \%$ of cellular users can establish communication with SINR from $-7 \mathrm{~dB}$ to $5.5 \mathrm{~dB}$. Moreover, from Figure $6 \mathrm{~b}$, it is seen that in all the communication scenarios, $60 \%$ of cellular users can establish communication with SINR from $10.5 \mathrm{~dB}$ to $18 \mathrm{~dB}$. As shown in Figures 5 and 6, our proposed scheme outperforms the RRA and RA w/o sectorization schemes by maintaining a specific distance between the D2D Rx and cellular users, as well as between the D2D Tx and Rx.

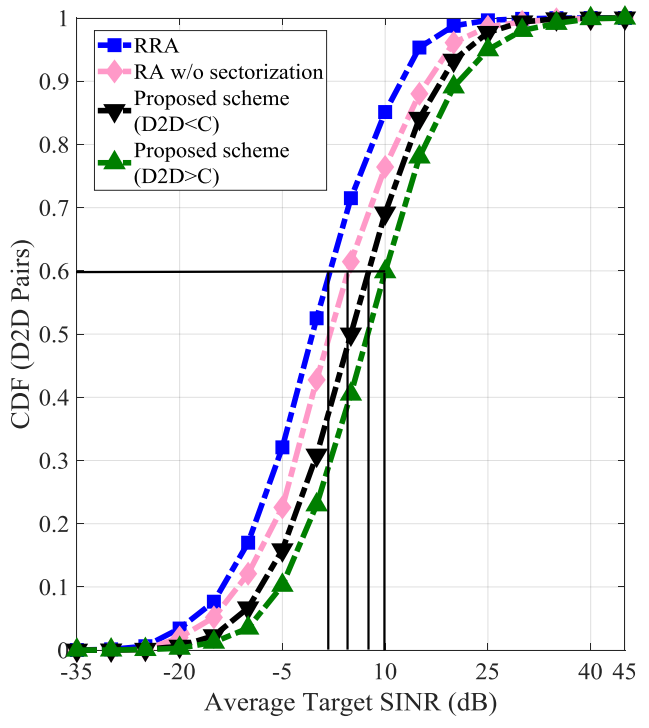

(a) for outer cell region

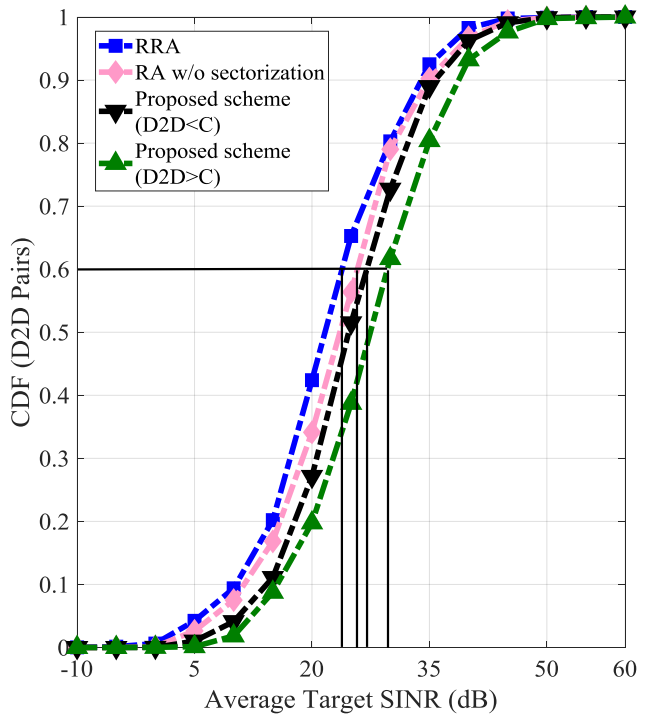

(b) for inner cell region

Figure 5. Average target signal-to-interference-plus-noise ratio (SINR) vs. cumulative distribution function (CDF) for D2D pairs, (a) outer cell region, (b) inner cell region.

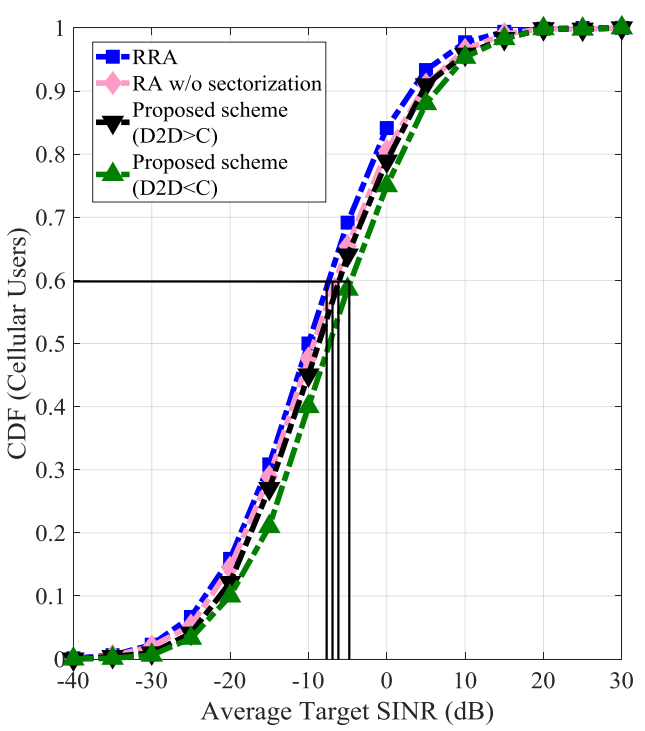

(a) for outer cell region

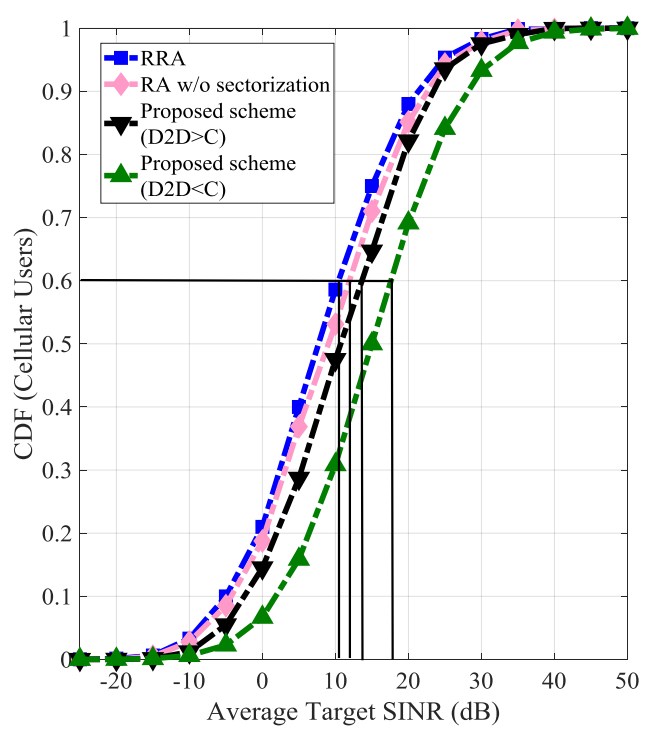

(b) for inner cell region

Figure 6. Average target SINR vs. CDF for cellular users, (a) outer cell region, (b) inner cell region. 
Figure $7 \mathrm{a}, \mathrm{b}$ and Figure $8 \mathrm{a}, \mathrm{b}$ show the SINR distributions of cellular users and D2D pairs by keeping D2D Tx and Rx in same and different locations in a cell, respectively. Figure 7a,b shows that for all the communication scenarios, $60 \%$ of cellular users can establish communication with SINR from $-7.5 \mathrm{~dB}$ to $-2 \mathrm{~dB}$ and $9 \mathrm{~dB}$ to $17 \mathrm{~dB}$, respectively. Moreover, Figure 8a,b shows that for all the communication scenarios, $60 \%$ of D2D users can establish communication with SINR from $2 \mathrm{~dB}$ to $18 \mathrm{~dB}$ and $23 \mathrm{~dB}$ to $28.5 \mathrm{~dB}$, respectively. Compared to the RRA scheme and RA w/o cell sectorization scheme, the proposed optmization method based on the location-based resource sharing scheme outperforms in terms if the SINR gain. These results validate the communication performance scenarios as explained above.

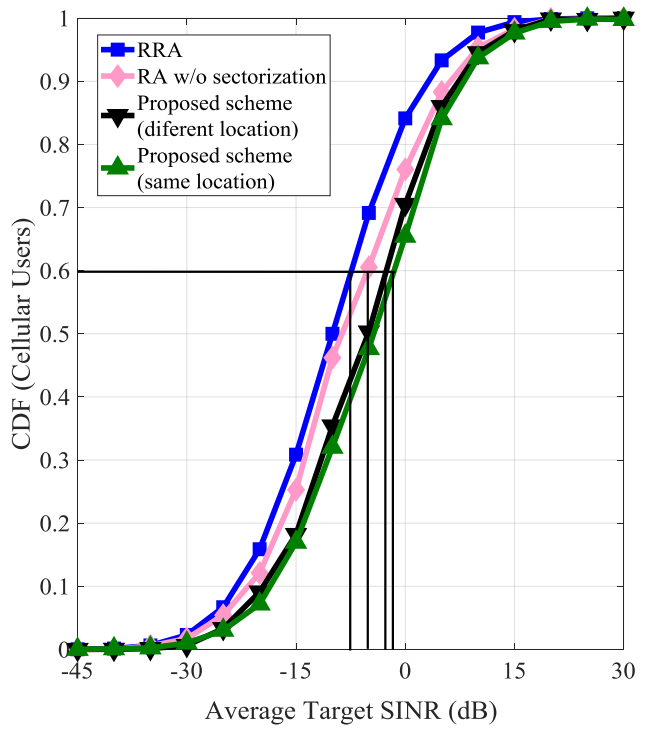

(a) for outer cell region

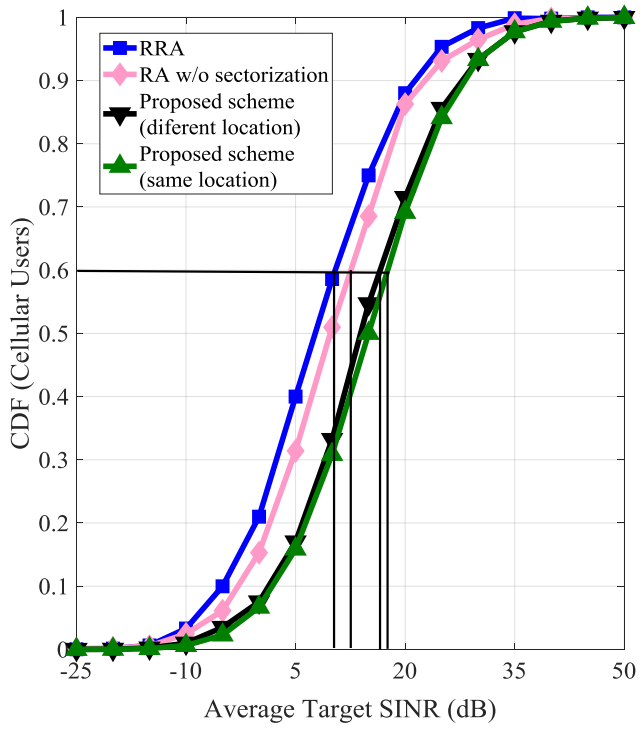

(b) for inner cell region

Figure 7. Average target SINR vs. CDF for cellular users with the same and different locations of D2D Tx and Rx, (a) outer cell region, (b) inner cell region.

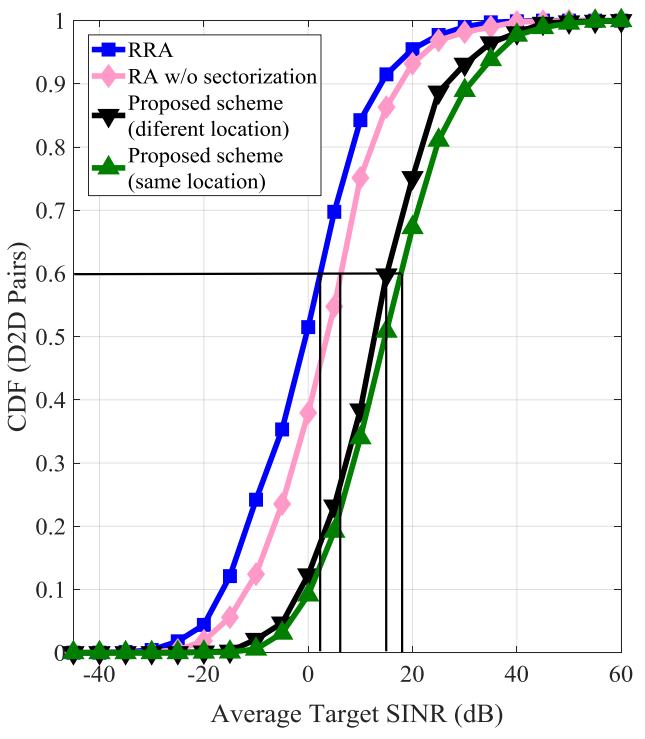

(a) for outer cell region

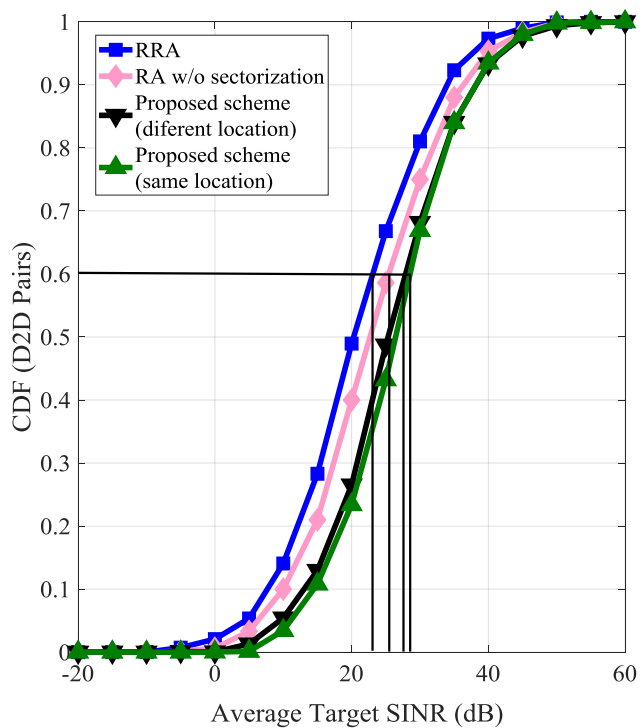

(b) for inner cell region

Figure 8. Average target SINR vs CDF for D2D pairs with the same and different locations of D2D Tx and $\mathrm{Rx},(\mathbf{a})$ outer cell region, (b) inner cell region.

Figure 9a-e shows the overall system throughput at the various distance between the D2D Tx and Rx. As shown in the figure, as the targeted D2D Rx is located far away from the Tx, the strength 
of the desired signal becomes weaker. Thus, the received power in the D2D Rx and average target SINR of the system reduces. It can be seen that when the D2D Tx and Rx are located in the same section, the co-channel interference is alleviated, thus a higher spectrum efficiency is gained. Therefore, our proposed scheme achieves better spectral efficiency than the traditional schemes proposed in the literature for different distances.

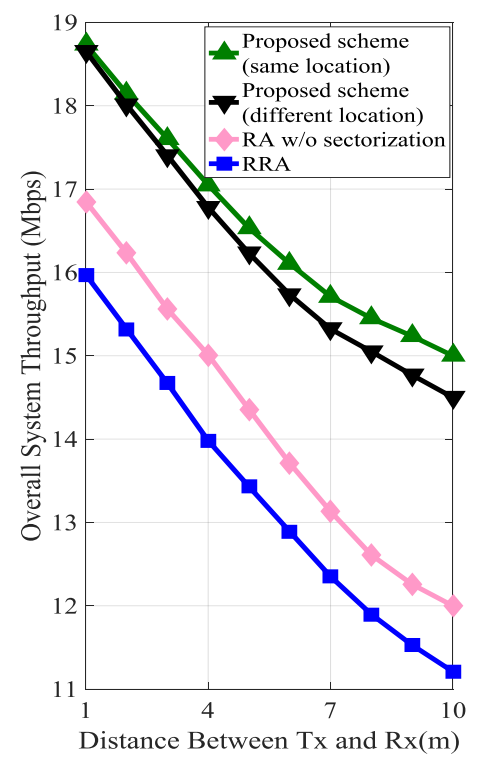

(a)

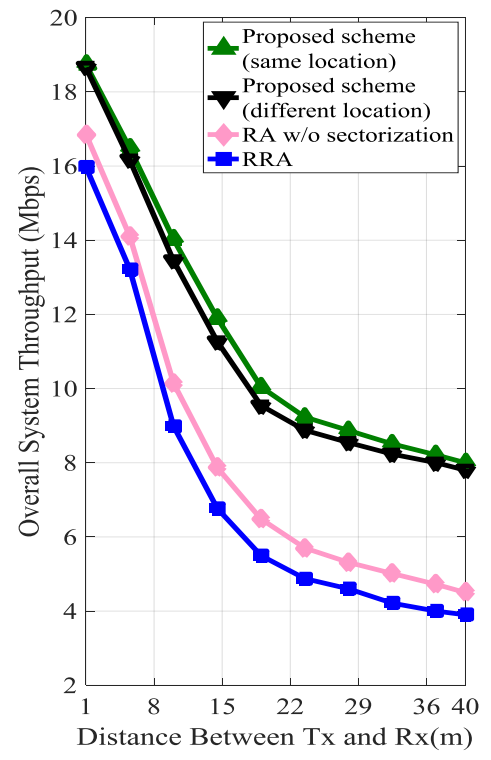

(d)

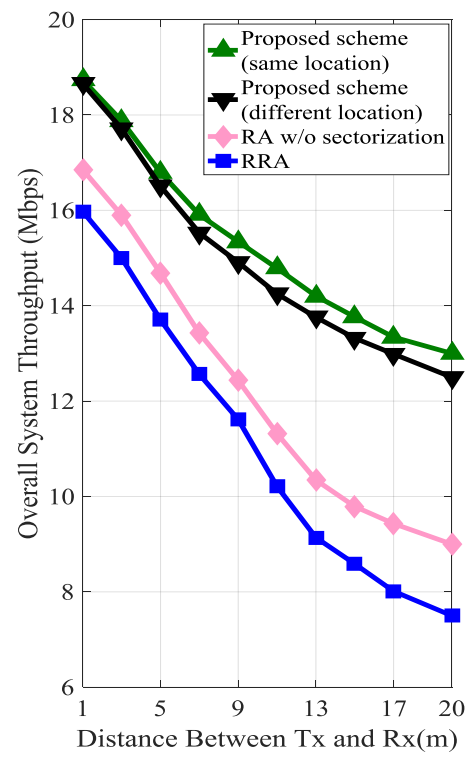

(b)

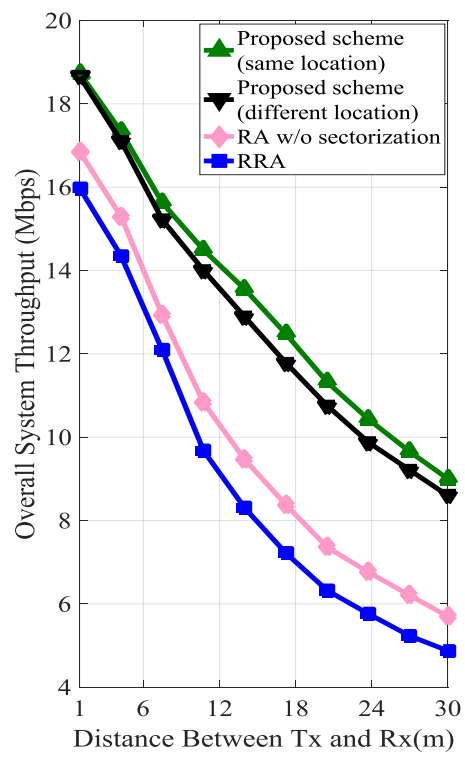

(c)

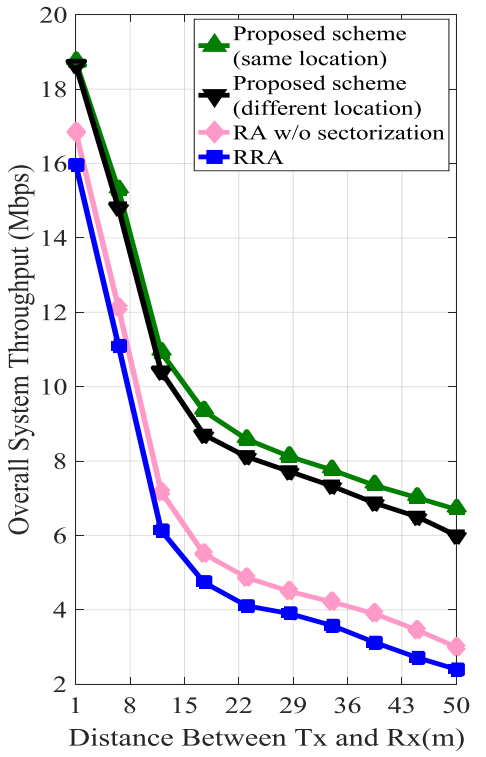

(e)

Figure 9. Overall system throughput with varying distance between D2D Tx and Rx. (a) 1-10 m, (b) 1-20 m, (c) 1-30 m, (d) 1-40 m, and (e) 1-50 m.

The average system spectral efficiency vs. CDF when both the D2D Tx and Rx are located in the same and different sections of the cell with varying number of D2D pairs as compared with cellular users are plotted in Figure 10a. As expected, the RRA scheme achieves minimum spectral efficiency. The Figure also shows that the system spectral efficiency in the proposed scheme with more number of D2D pairs than the cellular users in the same cell section is better than those in different cell sections. Therefore, the proposed scheme outperforms the RRA and RA w/o cell sectorization scheme. 
Figure 10b shows the overall cell throughput for various numbers of D2D pairs. The Figure clearly shows that the proposed scheme achieves higher throughput in comparison with the existing schemes. Unlike our proposed scheme, the RRA scheme does not utilize the entire frequency spectrum in each cell and more interferences occurred from the co-channel devices while the cellular resources are reused by the D2D pairs. However, the RA w/o sectorization scheme shows improved system throughput as compared to the RRA scheme.

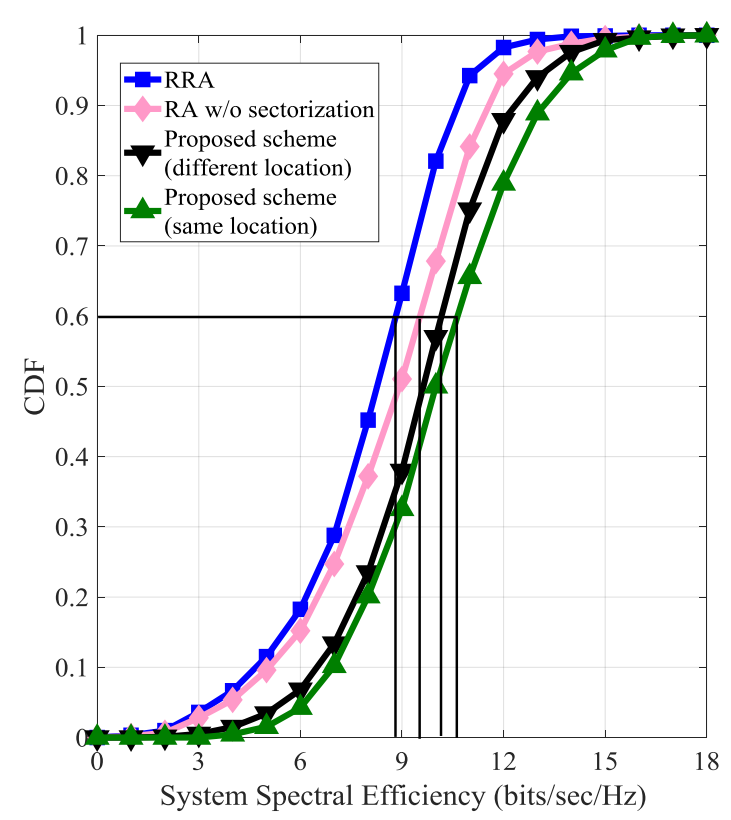

(a)

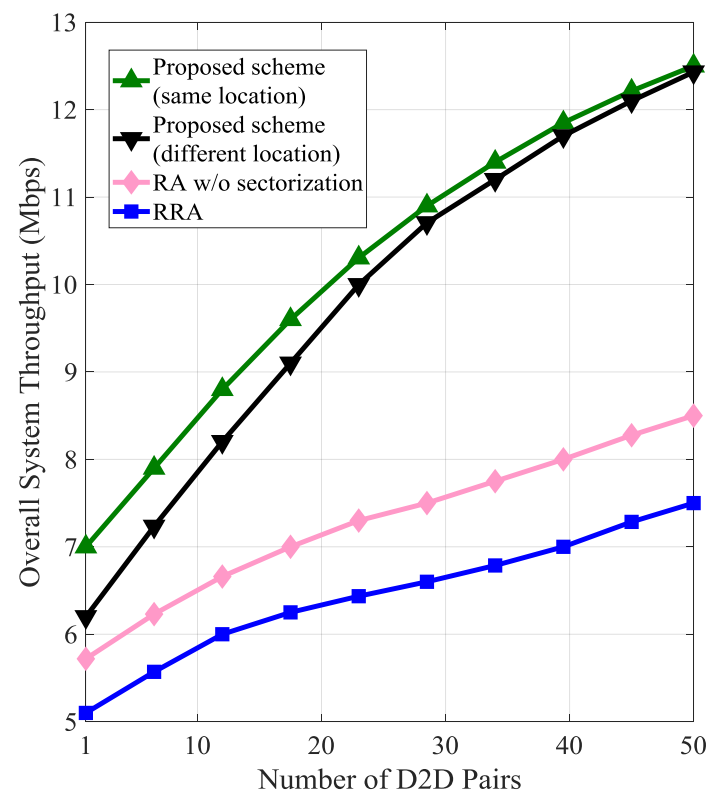

(b)

Figure 10. (a) System spectral efficiency vs. CDF and (b) overall system throughput with varying number of D2D pairs in the same and different locations.

\section{Conclusions}

D2D pairs reusing the uplink cellular resources in LTE-A multicell cellular networks using the FFR scheme are presented in this paper. The proposed scheme integrates the power control mechanism for both the D2D pairs and cellular users, which guarantees the effective calculation of the system outage probability in terms of their SINR. Based on our mathematical analysis, we guaranteed that the distance-based resource allocation and power-control scheme could support the QoS requirements of both the cellular and D2D pairs. This paper provides the network operators with less computation complexity, to reduce the interference between the D2D pairs, and between cellular users and D2D pairs. The simulation results show that the proposed scheme alleviates the overall system throughput and achieves higher spectral efficiency as compared to the existing schemes.

Author Contributions: D.D.N. organized and developed the proposal of the study, carried out the mathematical analysis, and performed simulations using Monte Carlo. Author S.S. provided guidance, key suggestions, and finalized the paper.

Funding: This research is partly supported by Basic Science Research Program through the National Research Foundation of Korea (NRF) funded by the Ministry of Education with Grant No. NRF-2015R1D1A1A01059962 and a part of the project titled 'Development of Automatic Identification Monitoring System for Fishing Gears', funded by the Ministry of Oceans and Fisheries, Korea with Grant No. 20170388.

Conflicts of Interest: The authors declare no conflicts of interest. 


\section{References}

1. Bojkovic, Z.; Bakmaz, B.; Bakmaz, M. Recent Trends in Emerging Technologies toward 5G Networks. In Proceedings of the 9th International Conference on Circuits, Systems, Signal and Telecommunications, Dubai, United Arab Emirates, 22-24 February 2015.

2. Duong, Q.; Shin, Y.; Shin, O.-S. Distance-based resource allocation scheme for device-to-device communications underlaying cellular networks. AEU Int. J. Electron. Commun. 2015, 69, 1437-1444. [CrossRef]

3. Zhao, W.; Wang, S. Low complexity power allocation for device-to-device communication underlaying cellular networks. In Proceedings of the 2014 IEEE International Conference on Communications (ICC), Sydney, NSW, Australia, 10-14 June 2014.

4. Ningombam, D.D.; Pyun, J.; Hwang, S.; Shin, S. Fractional frequency reuse scheme for interference mitigation in device-to-device communication underlying LTE-A networks. In Proceedings of the 2017 51st Asilomar Conference on Signals, Systems, and Computers, Pacific Grove, CA, USA, 29 October-1 November 2017.

5. Katsinis, G.; Tsiropoulou, E.E.; Papavassiliou, S. Joint Resource Block and Power Allocation for Interference Management in Device to Device Underlay Cellular Networks: A Game Theoretic Approach. Mobile Net. Appl. 2016, 22, 539-551. [CrossRef]

6. Lianghai, J.; Man, L.; Schotten, H.D. Context-aware cluster based device-to-device communication to serve machine type communications. In Proceedings of the 2017 IEEE International Conference on Communications Workshops (ICC Workshops), Paris, France, 21-25 May 2017.

7. Feng, D.; Lu, L.; Yuan-Wu, Y.; Li, G.Y.; Feng, G.; Li, S. Device-to-Device Communications Underlaying Cellular Networks. IEEE Trans. Commun. 2013, 61, 3541-3551. [CrossRef]

8. Kuruvatti, N.P.; Schotten, H.D. Post-resource sharing power allocation in cellular networks to coexist with D2D underlay. In Proceedings of the 2016 7th International Conference on the Network of the Future (NOF), Buzios, Brazil, 16-18 November 2016.

9. Katsinis, G.; Tsiropoulou, E.E.; Papavassiliou, S. On the Problem of Resource Allocation and System Capacity Evaluation via a Blocking Queuing Model in D2D Enabled Overlay Cellular Networks. In Int. Conf. on Ad-Hoc Net. and Wire; Papavassiliou, S., Ruehrup, S., Eds.; Springer: Cham, Switzerland, 2015; pp. 76-89.

10. Zhe, L. Performance Analysis of Network Assisted Neighbor Discovery Algorithms. Master's Thesis, Cardiff University, Cardiff, UK, 2012.

11. Katsinis, G.; Tsiropoulou, E.E.; Papavassiliou, S. On the Performance Evaluation of Distributed Resource Block and Power Allocation in D2D-enabled Multi-Cell Networks. In Proceedings of the 14th ACM Symposium on Performance Evaluation of Wireless Ad Hoc, Sensor and Ubiquitous Networks, Miami, FL, USA, 21-25 November 2017.

12. Ningombam, D.D.; Shin, S. Radio resource allocation and power control scheme to mitigate interference in device-to-device communications underlaying LTE-A uplink cellular networks. In Proceedings of the 2017 International Conference on Information and Communication Technology Convergence (ICTC), Jeju, Korea, 18-20 October 2017.

13. Sattiraju, R.; Klein, A.; Ji, L.; Zhou, C.; Bulakci, O.; Eichinger, J.; Kuruvatti, N.P.; Schotten, H.D. Virtual Cell Sectoring for Enhancing Resource Allocation and Reuse in Network Controlled D2D Communication. In Proceedings of the 2015 IEEE 81st Vehicular Technology Conference (VTC Spring), Glasgow, UK, 11-14 May 2015.

14. Kim, T.-S.; Lee, K.-H.; Ryu, S.; Cho, C.-H. Resource Allocation and Power Control Scheme for Interference Avoidance in an LTE-Advanced Cellular Networks with Device-to- Device Communication. Int. J. Control Autom. 2013, 6, 10.

15. Li, X.; Shankaran, R.; Orgun, M.A.; Fang, G.; Xu, Y. Resource Allocation for Underlay D2D Communication With Proportional Fairness. IEEE Trans. Veh. Technol. 2018, 67, 6244-6258. [CrossRef]

16. Zhang, Z.; Hu, R.Q.; Qian, Y.; Papathanassiou, A. D2D Communication Underlay in Uplink Cellular Networks with Fractional Power Control and Fractional Frequency Reuse. In Proceedings of the 2015 IEEE Global Communications Conference (GLOBECOM), San Diego, CA, USA, 6-10 December 2015.

17. Zhu, H.; Wang, J. Device-to-device communication in cellular networks with fractional frequency reuse. In Proceedings of the 2014 IEEE International Conference on Communications (ICC), Sydney, NSW, Australia, 10-14 June 2014. 
18. Sharma, S.; Gupta, N.; Bohara, V.A. OFDMA-Based Device-to-Device Communication Frameworks: Testbed Deployment and Measurement Results. IEEE Access 2018, 6, 12019-12030. [CrossRef]

19. Jiang, F.; Wang, B.-C.; Sun, C.-Y.; Liu, Y.; Wang, X. Resource Allocation and Dynamic Power Control for D2D Communication Underlaying Uplink Multi-Cell Networks. Wirel. Netw. 2016, 24, 549-563. [CrossRef]

20. Tehrani, M.N.; Uysal, M.; Yanikomeroglu, H. Device-to-device communication in 5G cellular networks: Challenges, solutions, and future directions. IEEE Commun. Mag. 2014, 52, 86-92. [CrossRef]

21. An overview of device-to-device communication in cellular networks. Available online: https: / / reader.elsevier.com/reader/sd/A6F2850A17C596B03F981C619FA194DEB33914A943A688 F4E45C913C154179879E4307972A65B27E22658F4E7BBDEEA5 (accessed on 14 August 2018).

22. Kim, J.; Karim, N.A.; Cho, S. An Interference Mitigation Scheme of Device-to-Device Communications for Sensor Networks Underlying LTE-A. Sensors 2017, 17, 1088.

23. Shah, S.T.; Gu, J.; Hasan, S.F.; Chung, M.Y. SC-FDMA-based resource allocation and power control scheme for D2D communication using LTE-A uplink resource. EURASIP J. Wirel. Commun. Netw. 2015, $2015,137$. [CrossRef]

24. Alouini, M.-S.; Goldsmith, A.J. Area spectral efficiency of cellular mobile radio systems. IEEE Trans. Veh. Technol. 1999, 48, 1047-1066. [CrossRef]

25. Li, X.; Wang, Z.; Sun, Y.; Gu, Y.; Hu, J. Mathematical Characteristics of Uplink and Downlink Interference Regions in D2D Communications Underlaying Cellular Networks. Wirel. Pers. Commun. 2017, 93, 917-932. [CrossRef]

26. Bąkowski, K.; Wesołowski, K.; Rodziewicz, M. Simulation Tools for the Evaluation of Radio Interface Technologies for IMT-Advanced and Beyond. In Simulation Technology in Networking and Communication; Pathan, A.-S., Monowar, M., Khan, S., Eds.; CRC Press: Boca Raton, FL, USA, 2014; ISBN 978-1-4822-2549-5.

(C) 2018 by the authors. Licensee MDPI, Basel, Switzerland. This article is an open access article distributed under the terms and conditions of the Creative Commons Attribution (CC BY) license (http://creativecommons.org/licenses/by/4.0/). 